\title{
The mechanobiology theory of the development of medical device-related pressure ulcers revealed through a cell-scale computational modeling framework
}

\author{
Adi Lustig ${ }^{1} \cdot$ Raz Margi $^{1} \cdot$ Aleksei Orlov$^{1} \cdot$ Daria Orlova $^{2} \cdot$ Liran Azaria $^{1} \cdot$ Amit Gefen $^{1}$ (1)
}

Received: 28 December 2020 / Accepted: 3 February 2021 / Published online: 19 February 2021

(c) Springer-Verlag GmbH Germany, part of Springer Nature 2021

\begin{abstract}
Pressure ulcers are localized sites of tissue damage which form due to the continuous exposure of skin and underlying soft tissues to sustained mechanical loading, by bodyweight forces or because a body site is in prolonged contact with an interfacing object. The latter is the common cause for the specific sub-class of pressure ulcers termed 'medical device-related pressure ulcers', where the injury is known to have been caused by a medical device applied for a diagnostic or therapeutic purpose. Etiological research has established three key contributors to pressure ulcer formation, namely direct cell and tissue deformation, inflammatory edema and ischemic damage which are typically activated sequentially to fuel the injury spiral. Here, we visualize and analyze the above etiological mechanism using a new cell-scale modeling framework. Specifically, we consider here the deformation-inflicted and inflammatory contributors to the damage progression in a medical device-related pressure ulcer scenario, forming under a continuous positive airway pressure ventilation mask at the microarchitecture of the nasal bridge. We demonstrate the detrimental effects of exposure to high-level continuous external strains, which causes deformation-inflicted cell damage almost immediately. This in turn induces localized edema, which exacerbates the cellscale mechanical loading state and thereby progresses cell damage further in a nonlinear, escalating pattern. The cell-scale quantitative description of the damage cascade provided here is important not only from a basic science perspective, but also for creating awareness among clinicians as well as industry and regulators with regards to the need for improving the design of skin-contacting medical devices.
\end{abstract}

Keywords Pressure injuries $\cdot$ Sustained mechanical loading $\cdot$ Cell and tissue deformations $\cdot$ Cell death $\cdot$ Inflammatory edema

\section{Introduction}

Pressure ulcers (PUs) are localized sites of tissue damage which form primarily due to the continuous exposure of skin and underlying soft tissues to sustained mechanical loading, typically over a bony prominence, by bodyweight forces or because a body site is in prolonged contact with an interfacing surface or object (Gefen et al. 2019). The latter is

Amit Gefen

gefen@tauex.tau.ac.il

1 The Herbert J. Berman Chair in Vascular Bioengineering, Department of Biomedical Engineering, Faculty of Engineering, Tel Aviv University, 6997801 Tel Aviv, Israel

2 School of Mechanical Engineering, Faculty of Engineering, Tel Aviv University, Tel Aviv, Israel the common cause for the specific sub-class of PUs termed 'medical device-related pressure ulcers' (MDRPUs), where the injury is known to have been caused by a skin-contacting medical device applied for a diagnostic or a therapeutic purpose (Levy et al. 2015, 2017b; Bader et al. 2019; Gefen et al. 2020).

During the outbreak of the coronavirus 2019 disease (COVID-19), there has been a considerable global rise in the number of patients who required an intensive care and are anesthetized and ventilated. Such patients, whether positioned prone due to an acute respiratory distress syndrome (ARDS) or ventilated supine, are constantly susceptible to PUs caused by the intensive use of medical devices on their skin surface, e.g., continuous positive airway pressure (CPAP) ventilation masks, tubing or pulse oximeters (Gefen 2020; Peko et al. 2020; Amrani and Gefen 2020; Gefen and Ousey 2020). Patients with 
breathing difficulties (typically infected by COVID-19 at the present time, but not exclusively those) are treated by means of the noninvasive CPAP ventilation therapy, as a first line of defense against development of ARDS. These typically immobile and often insensate or sedated intensive care patients are then experiencing continuous mechanical loads at the interface between the CPAP mask and the nasal bridge, a location known to be susceptible to tissue breakdown due to a MDRPU associated with use of a CPAP mask long before the outbreak of the COVID-19 pandemic (Fig. 1a) (Bader et al. 2019; Peko et al. 2020; Gefen et al. 2020). When the stiff CPAP mask is strapped to the head and applies sustained forces on a delicate (and small size) site like the dorsum of the nose, the thin layer of underlying soft tissues enclosed by the external device from one side and the bony prominence (i.e., the nasal bone) at the other side is highly susceptible to large tissue distortions that can potentially lead to deformationinflicted cell death (Gefen et al. 2019, 2020) (Fig. 2).
Specifically, etiological research has established that a thin layer of soft tissues subjected to sustained pressure and frictional forces (as in the case of the dorsum of the nose under the CPAP mask) is subjected to stress concentrations in the distorted tissues (Gefen et al. 2019, 2020). At the cell-scale, these stress concentrations translate to extreme cell deformations and in particular, to stretching of the plasma membrane (PM) of cells at the stress concentration sites, which leads to poration of the PMs of these cells (Gefen et al. 2019, 2020). Within less than an hour, that leads to loss of cell homeostasis in the affected cells, followed by apoptotic cell death (Slomka and Gefen 2012; Leopold and Gefen 2013; Gefen and Weihs 2016). Triggered by the initial cell death, nearby immune cells (e.g., macrophages, lymphocytes and mast cells) as well as keratinocytes, fibroblasts and endothelial cells secrete cytokines to initiate the immune response and prepare the ground for the arrival of tissue-repairing cells (Van Linthout et al. 2014). In the context of PU etiology (including
Fig. 1 Finite element (FE) microscale modeling of the subcutaneous adipose tissue of the bridge of the nose subjected to the large deformations caused by the use of a noninvasive ventilation mask: a A full-thickness pressure injury developed at the bridge of the nose [reproduced with permission from (Black et al. 2015)]. b Cross-sectional anatomy of the nose, showing the region of subcutaneous adipose tissue which is compressed between a continuous positive pressure airway (CPAP) mask and the nasal bone. $\mathbf{c}$ Model geometry and the applied twostep loading regime downward deformation to simulate compression by the CPAP followed by normal pressure loads to simulate the formation of edema
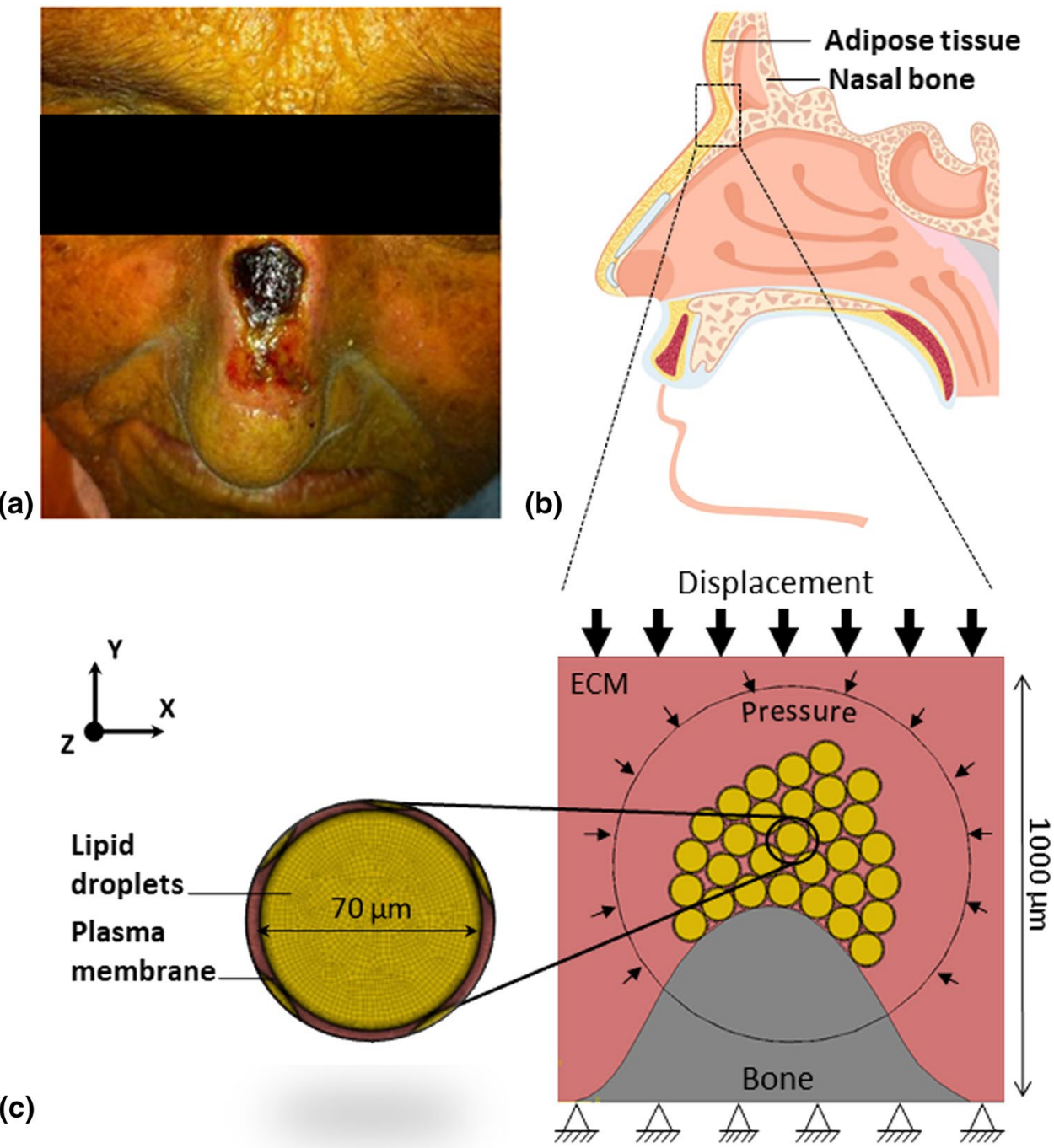
Fig. 2 The mechanobiological factors contributing to cell death and tissue damage in a forming pressure ulcer: (i) direct deformation exposure that damages the cytoskeleton and plasma membrane of cells, thereby compromising their ability to control transport through the plasma membrane; (ii) inflammatory edema; and (iii) ischemia. The combined effect of these three contributors to cell and tissue damage explains the nonlinear nature of the cumulative damage and the rapid damage progression in pressure ulcers

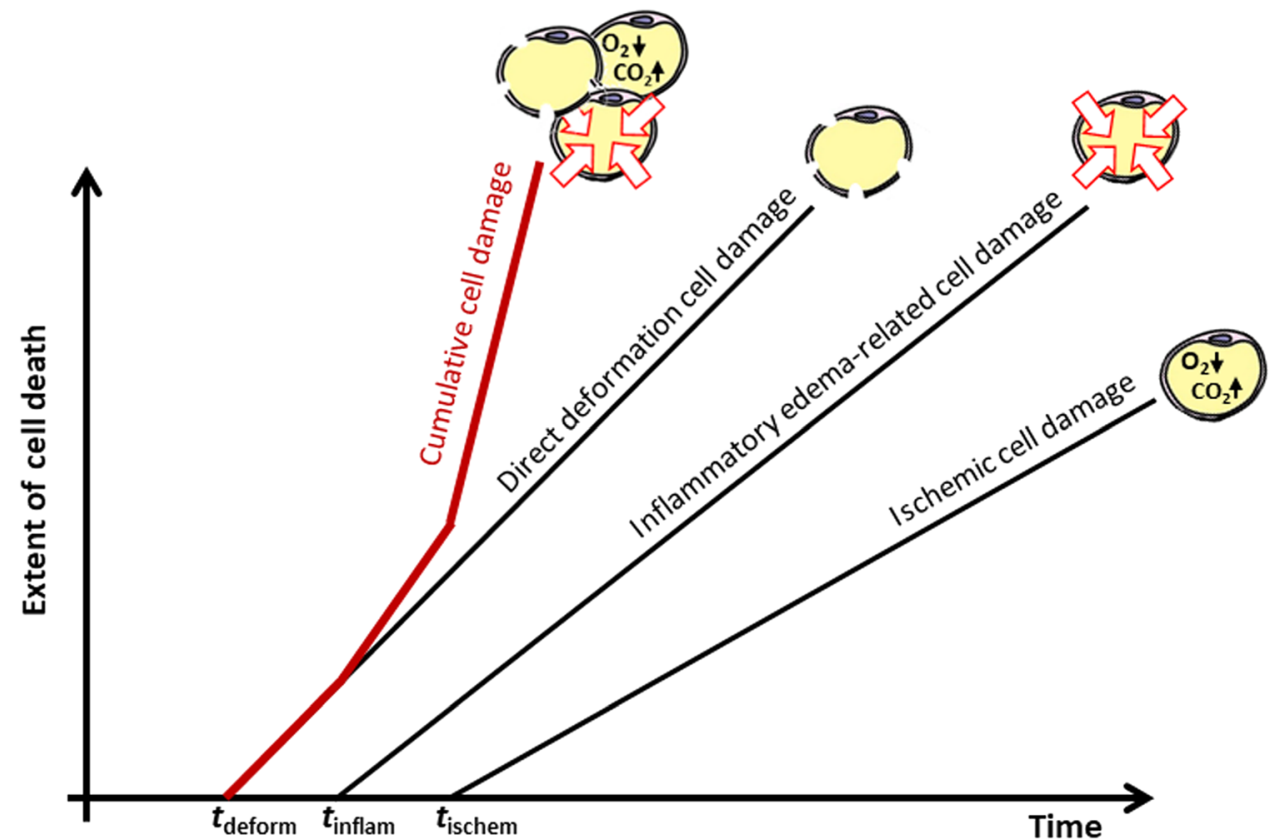

the etiology of MDRPUs), however, the inflammatory signaling itself is a potential contributor to additional soft tissue damage (Gefen 2018, 2020; Gefen et al. 2019, 2020). This is because the secreted pro-inflammatory cytokines act to increase the vascular permeability around the site of the initial cell death, to facilitate migration of large number of leukocytes which normally circulate in the vasculature, to the location of the mechanically induced damage (Gefen 2018, 2020; Gefen et al. 2019, 2020). While this vascular extravasation facilitates the infiltration of numerous leukocytes to the initial cell death site, it also results in leakiness of the vasculature, leading to accumulation of plasma fluids in the interstitial spaces, which gradually builds up a localized edema. Since the affected soft tissues are nearly unable to expand in volume within the confined space between the stiff CPAP mask and nasal bone surfaces as the edema develops, the localized interstitial pressures must rise sharply, causing additional deformation of (more) cells. The inflammatory system may then release reactive oxygen and nitrogen species to degrade the extracellular matrix (ECM), in an attempt to relieve the rising interstitial pressures, which further exacerbates the tissue damage, now extracellularly (Gefen 2018, 2020; Gefen et al. 2019, 2020). When the interstitial pressures reach a level that obstructs the vasculature itself, the damage cascade evolves to the next stage, where the poor blood perfusion into the affected tissues induces hypoxia and ischemia. These three key contributors to PUs, namely direct cell and tissue deformation, inflammatory edema and ischemic damage are therefore activated sequentially to fuel the injury spiral and worsen the state of tissues altogether, with a combined effect (Gefen 2018, 2019; Gefen et al. 2019).

Here, we visualize and analyze the above etiological mechanism using a new cell-scale modeling framework, which considers the deformation-inflicted and inflammatory contributors to the damage progression in a DRPU under a CPAP mask at the microarchitecture of the nasal bridge tissues. We simulated the occurrence of these two damage steps using a cause-and-consequence form, which depicts the catastrophic micro-damage progression that underpins the formation of MDRPUs.

\section{Methods}

The computational finite element (FE) modeling framework described below has been developed to achieve the following aims: (i) Evaluate the mechanical loads that are formed in the microscale of subcutaneous adipose tissue of the bridge of the nose, while this site is exposed to external large deformations due to use of a CPAP mask. (ii) Investigate the possible occurrence of cell death associated with inflammatory edema and the subsequent rise in the interstitial pressure in the deformed tissue. (iii) Compare the cell death propagation rates induced by the deformation-only versus the combined loading of direct deformation with the edema-related pressure. To achieve the above aims, we have considered a group of adjacent adipocyte cells subjected to large deformations, in which cell viability is subjected to a cell-death prediction model that accounts for the magnitude of the strains experienced by the PM in each cell over time (Gefen et al. 2008). 


\subsection{Geometry and mechanical properties}

We developed variants of a FE model of adipose tissue at a microscale, which includes 30 mature adipocytes in proximity to a bony prominence surface simulating the nasal bone (Fig. 1b). The group of adipocyte cells were surrounded by ECM (Fig. 1c) for uniform delivery of external tissue deformations to the cell structures, in order to eventually simulate the resulting cell death events. The adipocytes were made uniform in size, with a $70 \mu \mathrm{m}$ diameter and each included a $1 \mu \mathrm{m}$-thick plasma membrane (PM) (Fig. 1c) (Comley and Fleck 2010). The overall model dimensions (including the ECM component) were $1000 \times 1000 \mu \mathrm{m}$ (Fig. 1c). The model geometry (Fig. 1c) was created using the SolidWorks® 2019 software package (ver. 2019, Dassault Systems Simulia Corp., Johnston, RI, USA).

Each of the aforementioned model components excluding the bone was assumed to behave as an isotropic hyperelastic compressible material that obeys a Neo-Hookean strain energy density function $W$ (Breuls et al. 2002) (Ben-Or Frank et al. 2015):

$W=\frac{k}{2}\left(J_{3}^{1 / 2}-1\right)^{2}+\frac{G}{2}\left(J_{1}-3 J_{3}^{1 / 3}\right)$

where $k$ is the bulk modulus and $G$ is the shear modulus. The parameters $J_{1}$ and $J_{3}$ denote the first and third invariants of the left Cauchy-Green deformation tensor. Bone tissue (Fig. 1b) was modeled as isotropic and linear elastic (Katzengold et al. 2016). The $k$ and $G$ parameter values for each model component have been selected based on published literature, as detailed in Table 1. "Tie" constrains were applied at all material interfaces.

\subsection{Boundary and loading conditions}

The FE model variants were solved considering that all cells were in a force and moment equilibrium, yielding that $\nabla \sigma_{\mathrm{ij}}=0$, where $\sigma_{\mathrm{ij}}$ is the Cauchy stress tensor and $t_{i}=\sigma_{\mathrm{ji}} n_{j}$ are the applied tractions of force or pressure (as detailed

Table 1 Mechanical properties of the model components

\begin{tabular}{llll}
\hline Model component & $k$ & $G$ & $\begin{array}{l}\text { Number } \\
\text { of ele- } \\
\text { ments }\end{array}$ \\
\hline Bone $^{\mathrm{a}}$ & $5812(\mathrm{MPa})$ & $2682(\mathrm{MPa})$ & 7722 \\
Lipid droplets $^{\mathrm{b}}$ & $20(\mathrm{kPa})$ & $2.07(\mathrm{kPa})$ & 80,381 \\
Plasma membrane $^{\mathrm{b}}$ & $24.16(\mathrm{kPa})$ & $2.5(\mathrm{kPa})$ & 53,698 \\
Extracellular matrix $^{\mathrm{b}}$ & $0.69(\mathrm{kPa})$ & $0.18(\mathrm{kPa})$ & 62,547 \\
\hline
\end{tabular}

a (Katzengold et al. 2016)

b(Ben-Or Frank et al. 2015) below), for which $n_{j}$ denotes the unit vector normal to the cell surfaces (Fig. 1c).

We set fixed boundary conditions to the bottom and side surfaces of the model for all translations and rotations (Fig. 1c) to facilitate unidirectional loading from the top surface, simulating the directional compressive loading from the CPAP mask onto the nasal bridge. To simulate this initial external deformation and the following inflammatory edema, we employed a two-staged loading regime: (i) a uniformly distributed force applied on the upper model surface, with magnitude equivalent to a compressive strain of $55 \%$ in the simulated soft tissue domain, and (ii) a compressive pressure of $1.33 \mathrm{kPa}$ applied normally to an imaginary circle surrounding all the adipocytes (Fig. 1c); this pressure magnitude representing a regionally elevated interstitial pressure level due to edema (Michel and Curry 1999; Husmann et al. 2006). Noteworthy is that applying the pressure on the aforementioned imaginary circle that circumferences of the group cells were found (in preliminary simulations) to have an effect on cell deformations that was indistinguishable from the effect observed when pressures were applied normally, separately onto each individual cell. The loading was increased stepwise, in a manner that allowed to separately increase the external or the internal (edema-related pressure) loads, for the purpose of studying these effects on the cell loading states in isolation from each other.

\subsection{Numerical methods and outcome measures of finite element model}

The meshing and all subsequent FE analyses were conducted using the ABAQUS software suite (ver. 2020, Dassault Systems Simulia Corp., Johnston, RI, USA). The element type was CPS4R; the number of elements for each model component is further listed in Table 1. We have calculated effective logarithmic (true) strains and (von Mises) stresses in the PMs of each of the modeled adipocytes. To account for any potential edge effects, we only considered the calculated strain and stress data from the 15 adipocytes that reside in the center of the cell group (out of the total of 30 cells, i.e., we did not consider strain/stress data from cells directly bordering the ECM; Fig. 1c). Outcome measures included the distribution of strains and stresses in the above 15 central cells and the peak strain/stress values at the PMs.

\subsection{Strain-time cell-death threshold model}

In order to define when the death of a certain cell occurs in our FE simulations, we have used the injury threshold law first suggested by Linder-Ganz et al. (2006) (Linder-Ganz et al. 2006), based on histological studies of animal tissues subjected to sustained deformations. We have used a specific formulation of this injury threshold, named the 'Gefen 
curve' (Gefen et al. 2008; Gefen 2019), which describes the time-dependent critical (i.e., lowest) strain values that are most likely (i.e., at a 95\% probability) to cause deformationinduced cell death (caused by damage to cytoskeletal and PM structures), as follows:

$E^{c}(t)=\frac{K}{1+e^{a\left(t-t_{0}\right)}}+C$

where $K, t_{0}, a$, and $C$ are empirically-determined constants that are specified in (Gefen et al. 2008) and $t$ is the duration of tissue exposure to the sustained strain level after the initial exposure time point $t_{0}$. We used the aforementioned cellscale injury threshold to identify cells in our FE simulations that at a certain time point would experience critical strains at their PMs. As a result, we could also attribute survival times to the remaining cells in each calculation step. In the first step, we substituted the maximal PM strain value (per cell), obtained at the initial loading stage where only external deformation was considered. If a PM segment of a certain cell exceeded the strain-time injury threshold defined in (Gefen et al. 2008) at this stage, it was marked as 'dead' at the time where the threshold level has been exceeded, and that specific cell was then excluded from the further simulation steps (hence, that dead cell did not participate in the load sharing with the other cells from that point in time onwards). In addition, once cells have died, we activated the loading associated with the inflammatory edema. This process in practice resulted in four loading cycles after which, only part of the cells have 'survived'. We have also conducted a series of simulation cycles where we did not activate the pressure from edema, to study the effect of edema in isolation from the effect of direct deformation on the simulated cell viability. To account for the variability in the critical strain levels associated with specific tissues of individuals and in order to test the sensitivity of our present modeling to this characteristic, we employed two additional sets of data-driven formulations based on the 'Gefen curve' (Gefen et al. 2008; Gefen 2019), with critical strain levels that were reduced by $20 \%$ and $30 \%$ with respect to the original curve.

\section{Results}

Distributions of effective stresses in the adipocyte group prior to and following the first deformation-induced cell death event and with the addition of the pressure associated with inflammatory edema, are shown in Fig. 3a-c. Before the edema has been triggered, the effect of the first death event of a single cell on the stress exposures in the other cells was particularly dominant for the cells in the direct vicinity of the dead cell and moreover, for the ones near the bony surface. The more peripheral cells were mildly affected. Once the edema has been introduced, the loading levels increased within all the cells but again, more profoundly, in the cells adjacent to the bony surface and among these cells, in the cells in the direct proximity to the location of the dead cell (Fig. 3c). The volumetric exposures of the PMs in the 15 adipocytes after the two-staged loading regime (first just direct deformation, then deformation plus the pressure of edema) were analyzed for all the 15 centered cells (Fig. 3d) and also, for a one selected neighbor cell to a dead cell (Fig. 3e). The shift of the PM stress curves to the right-hand side post application of the edema pressure is evident, indicating the considerable increase in cell stress exposures caused by the added edema pressure. These stress exposure histograms shown in Fig. 3d, e likewise demonstrate that the deformation-induced single cell death event had a stronger local effect on a neighboring cell (Fig. 3e) than on the entire cell group (Fig. 3d), but the pressure rise due to the edema had both localized and regional effects (Fig. 3d, e).

The strain distributions in the adipocyte group after each of four simulation steps are shown in Fig. 4. Following the first loading step of pure deformation, the highest PM strains are found in cells near the bony prominence, causing abovecritical PM strains in one of the cells adjacent to the bone surface. The outcome of this simulation step is therefore that this one cell had ceased to take part in the loadbearing and has been marked as 'dead' for subsequent simulation steps. Also, as this death event has triggered the edema response, from this point onwards, we applied the combined loading of direct deformation (associated with the CPAP mask) superimposed with the interstitial pressure that had built up from the edema and the confinement on tissue expansion at that site (as the tissue at this region is considered to be trapped between the CPAP mask and the nasal bone). This combined loading resulted in elevated and above-critical strains at the PMs of neighboring cells to the locations of dead cells, due to the absence of cells that had previously participated in the loadbearing and have died and thereby, ceased to share the loading. The maximal PM strains consistently appeared in cells near the bone surface and in those cells, at PM segments that were either facing the bone tissue or in direct contact with neighboring cells (magnified views are included in Fig. 4).

A number of dead cells for pure deformation versus the combined direct deformation and inflammatory edema loading over time, for three sets of data-driven strain-time cell injury thresholds, are shown in Fig. 5. The cumulative effect and nonlinearity of the progression of cell death with the introduction of the inflammatory edema loading step in the modeling are well demonstrated in these plots. The data in Fig. 5 also exemplifies how cell-death thresholds of individuals may affect the onset and progression of cell death 
Fig. 3 The effective stress distribution prior to (a) and following (b) the first deformationinduced cell death event. Once edema develops, the loading levels increase in all cells but more profoundly, in the cells adjacent to the bony surface and among these cells, in the cells in direct proximity to the location of the dead cell (c). The volumetric exposure of plasma membrane elements to effective stress in all the 15-centered cells (d) and in one selected neighbor cell to the first dead cell (e), the location of which is marked using arrows in frames a,b,c
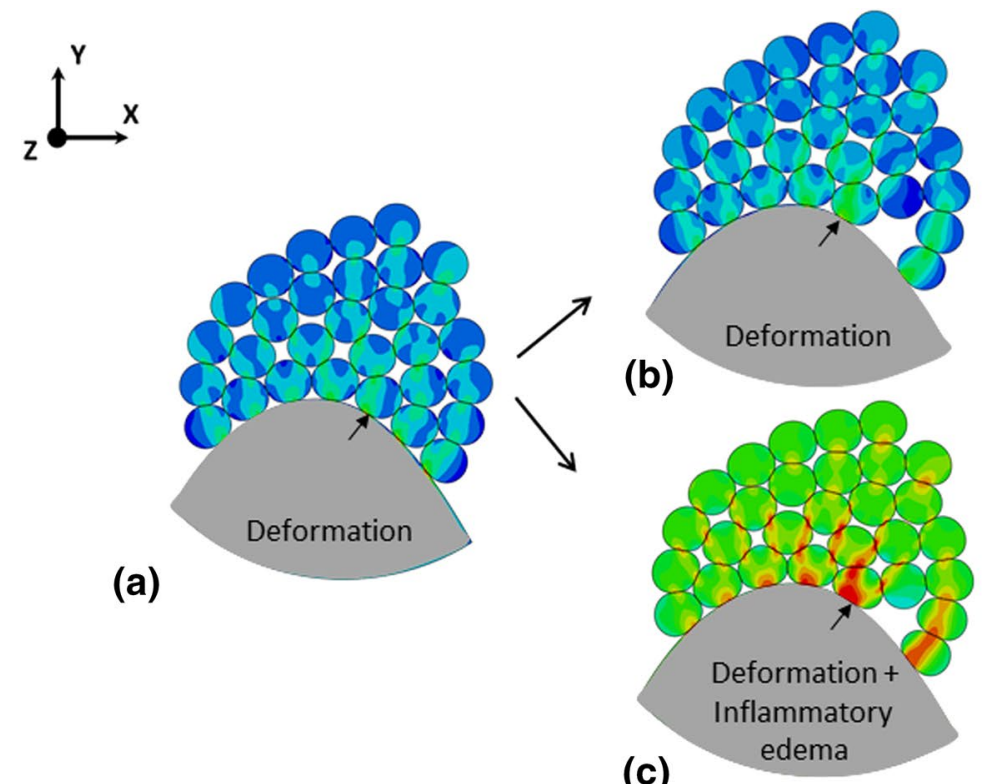

Effective

Stresses

[kPa]

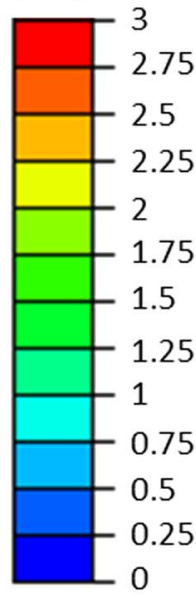

(a)

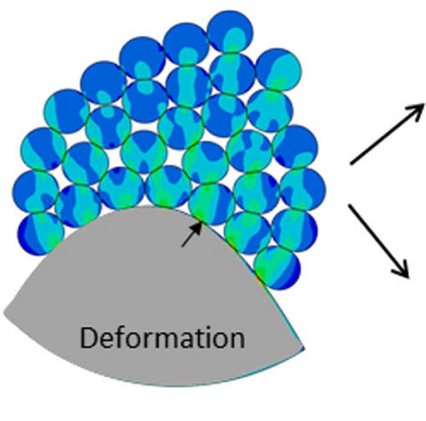

(c)

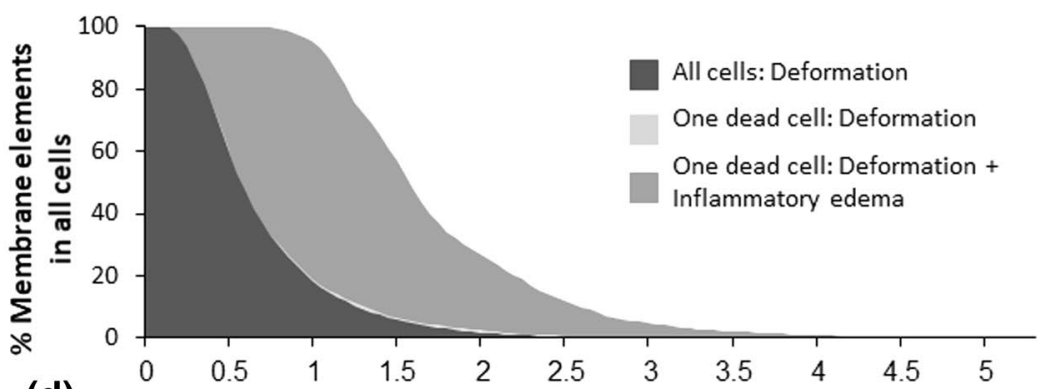

(d)

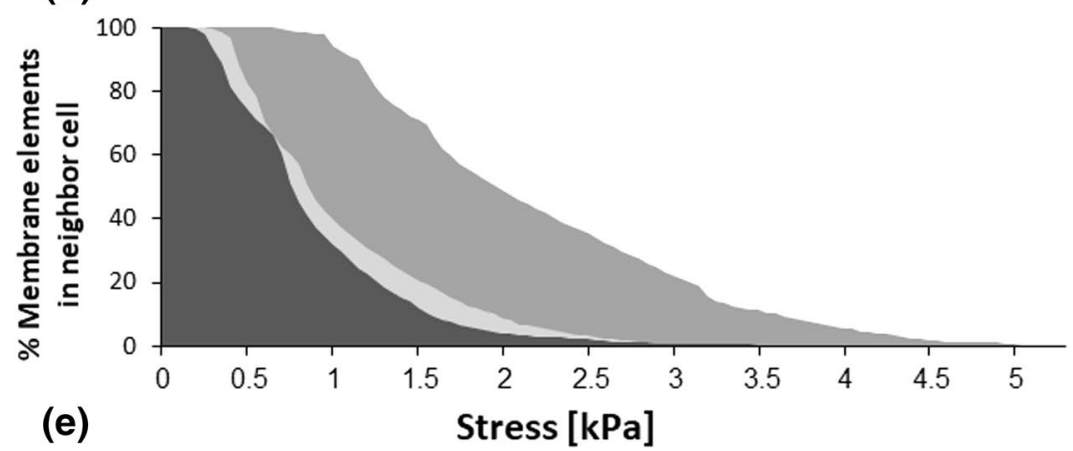

in a forming PU. Specifically, using the original formation of the 'Gefen curve' (as in the Gefen et al. 2008 paper), the simulations predicted that 5 cells would die after approximately 385 min (Fig. 5a) but $20 \%$ or $30 \%$ reduction in the cell injury tolerance had shortened that timeframe to just 175 min (Fig. 5b) or $141 \mathrm{~min}$ (Fig. 5c), respectively. Also, after $300 \mathrm{~min}$, the latter two simulation sets already resulted in approximately twice the extent of cell death than where the cell injury curve was at its baseline level.

To summarize these computational results, the cells that are most susceptible to both deformation-induced and inflammatory-related cell damage are the ones near the bone surface (Fig. 3); the rise in interstitial pressure with onset of the inflammatory edema accelerates the cell damage regionally and each dead cell implies that surrounding cells are subjected to increased loading (Fig. 4), in a progressive injury cascade (Fig. 5).

\section{Discussion}

In the present work, we investigated the mechanobiology of PU onset and development, with a focus on the cellscale biomechanics leading to MDRPUs, using the CPAP 


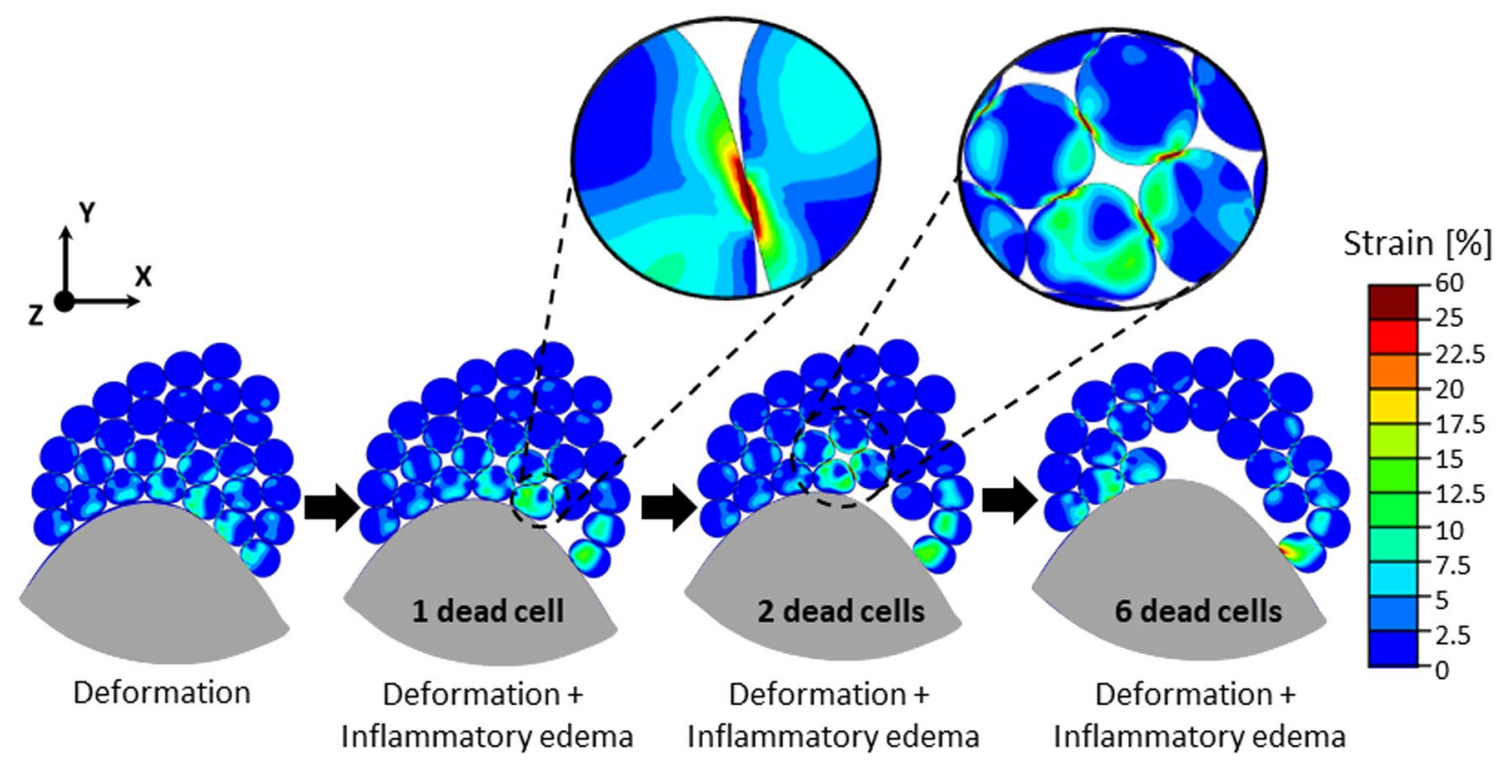

Fig. 4 Simulation of progressive damage in the cell group: We used the Gefen et al. (2008) cell-scale injury threshold to identify cells in our finite element simulations that at a certain time experience critical strains to their plasma membranes. If a plasma membrane segment of a certain cell exceeded the strain-time injury threshold, it was marked

ventilation scenario as a typical example. Triggered by the direct deformation associated with the use of noninvasive ventilation masks (Peko Cohen et al. 2019), facial soft tissues at the nasal bridge undergo considerable distortions that lead to deformation-inflicted cell death. This initial damage activates the innate immune response which in turn causes edema that, under these specific circumstances of a small soft tissue region confined between the CPAP mask and skull bone, elevates the interstitial pressures and thereby contributes to the progressive cell death. By means of cellscale computational modeling combined with data-driven strain-time cell injury thresholds, we visualized and quantitatively demonstrated the evolution of this vicious cycle considering the primary deformation and secondary inflammatory damage factors (but not the tertiary ischemic damage factor). Despite that the hypoxic and acidic conditions expected to be caused by ischemia were not considered here, we were able to demonstrate the nonlinearity and cumulative nature of this cell-level vicious cycle process, which is exemplified in this work through computational modeling for the first time in the literature.

The present simulations revealed that the cells adjacent to the bony surface are the most likely to die first in such a MDRPU scenario, due to the adipose-bone stiffness gradients leading to the most elevated PM strains in cells at this location (Gefen 2020). The PM is a relatively stiff cell structure which is reinforced by proteins of the cytoskeleton to maintain the cell shape and resist external loads (Shoham et al. 2014; Katzengold et al. 2015). This implies that as 'dead' at the relevant time point and that specific cell ceased to take part in the loadbearing and was excluded from the further simulation steps. Additionally, once cells have died due to the deformation exposure, we activated the additional pressure loading associated with the inflammatory edema

cell-cell contacts, which are typical to subdermal adipose tissue (Shoham et al. 2015, 2017), would also induce intracell stress concentration sites at the microscale, as indeed demonstrated in the present modeling. The proximity to the bony surface and the cell-cell contacts superimposed cellscale stress concentrations, hence making the cell layers near the bone more susceptible to both the direct deformation damage and the inflammatory damage that followed. Remarkably, however, once edema was introduced, cell stresses grew regionally from the locality of the bone surface, but still the cells that were nearest to the bone were the most influenced (Figs. 3,4).

Contrarily to the effect of edema, each dead cell (near the bone surface) had a localized effect on stresses in cells that directly neighbor the dead cell which ceased from participating in the load sharing, but the effects of the absent cell did not considerably project to cells that were farther than the direct vicinity. Nevertheless, each missing (dead) cell was sufficient to cause such increase in the loading state in adjacent cells that was predicted to cause mechanical damage to the PMs of these neighboring cells as well, thereby escalating the damage and progressing the injury spiral. In a living system, this would further trigger activity of the immune system through cytokine signaling, which would continue to buildup the edema and cause more secondary cell and tissue damage. Importantly, given that each dead cell in the modeling (or in reality) has at least 4-5 neighboring cells that are forced to take more of the external loading (induced by the CPAP mask straps in this case), once a dead 
Fig. 5 The nonlinear effect of the commutative damage caused by direct deformation combined with inflammatory edema, demonstrated through the number of dead cells for pure deformation versus the combined direct deformation and inflammatory edema loading over time, for 3 sets of data-driven strain-time cell injury thresholds: The original 'Gefen curve' a the aforementioned curve with the critical strain level reduced by $20 \%$ b and; $30 \%$ c. The straintime injury thresholds used as inputs for each set of simulations are presented in gray in the top left-hand side of each plot of results. Red arrows indicate when 5 cells have died
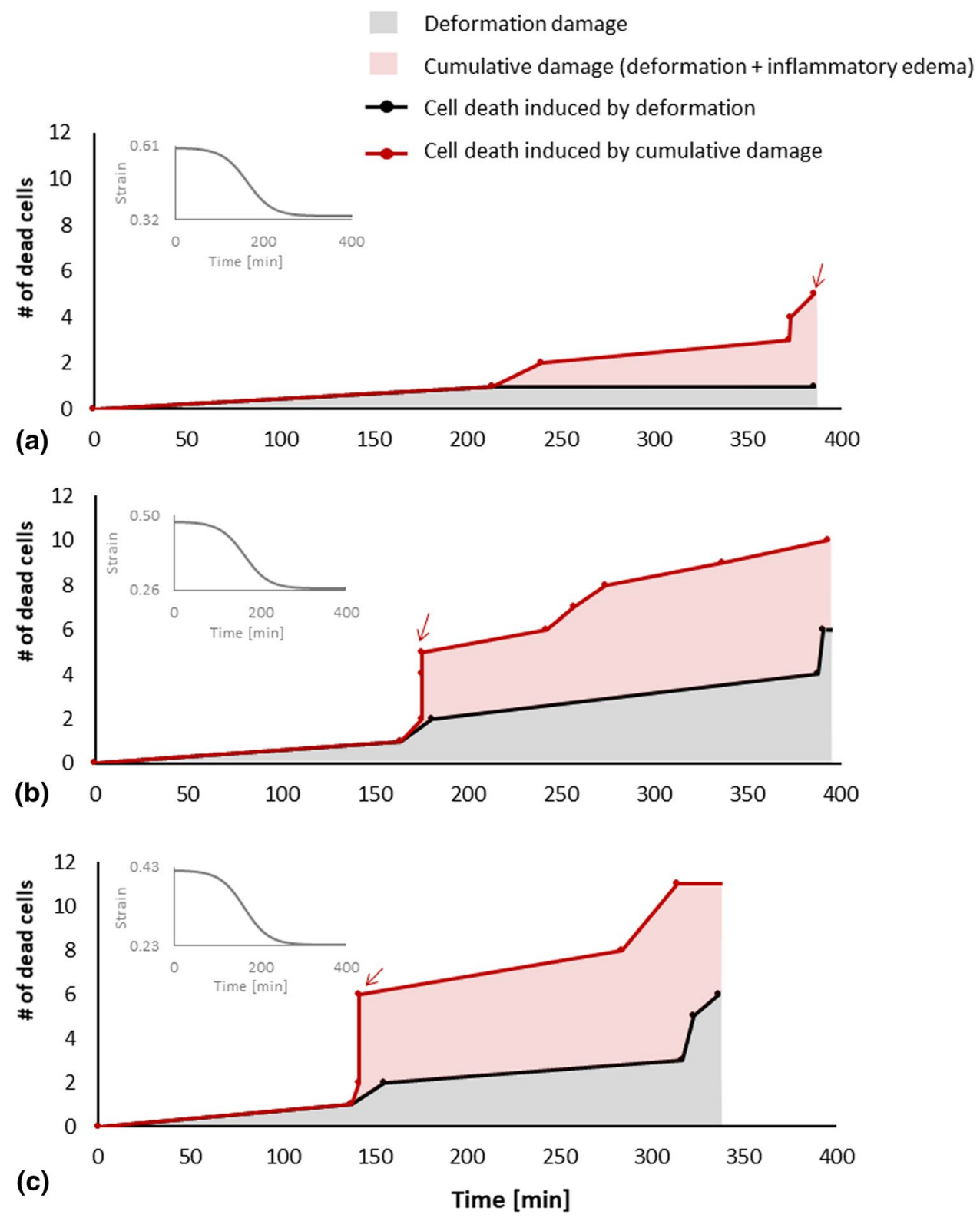

cell has terminated its participation in the load sharing, the nonlinearity and accelerating nature of the damage progression are to be expected. This is indeed, demonstrated in our presently reported data.

Due to the variability in individual anatomies, cell and tissue tolerances to loading and other confounding factors, it is not possible to determine generic quantitative values for cell and tissue damage thresholds in individuals as a function of the load and exposure time per se (Gefen et al. 2008; Gefen 2018, 2019). Nevertheless, we were able to demonstrate here that a lower cell-level injury threshold would inevitably lead to a greater extent of cell damage which ultimately presents itself as a more severe macroscopic presentation of the MDRPU (Fig. 1a). Our numerical simulation results further reinforce the theory of nonlinearity of the cumulative damage in PU (including MDRPU) formation (Gefen 2018, 2019; Gefen et al. 2019). In particular, the present work demonstrates the detrimental effects of exposure to a high level of tissue and cell strains, which will activate deformation-inflicted damage in the affected cells almost immediately, thereby inducing inflammatory edema which will exacerbate the cell-scale mechanical loading conditions and thereby progress the cell damage further, causing clinically meaningful tissue damage (Fig. 1a) in relatively short times.

As with any modeling work, limitations are inevitable and should be discussed here for completeness. Firstly, we did not consider the third contributing factor to the damage cascade, which is the ischemic damage (Gefen 2018, 2019). This can be considered in future work but would 
require transition to a multiphysics modeling framework, accounting for the anticipated interactions of the mechanical state of cells with nutrient mass transport and oxygen diffusion in the model domain, as opposed to the purely structural analyses conducted here. Secondly, we did not consider effects that could be characteristic to the individual such as anatomy (e.g., sharper bony surface), or excessive strapping of the CPAP mask leading to greater tissue and cell deformations at baseline, or any protective measures such as prophylactic dressings that would alleviate the localized cell deformations (Peko Cohen et al. 2019). Lastly, the modeling can be extended to larger populations of cells, which would allow simulations that extend from the microscale to the macroscale, or a complete hierarchical modeling approach can be pursued. While these features are interesting to include from a scientific and visualization perspectives, they do not appear to offer immediate additional value in terms of understanding the fundamental problem of MDRPUs and mitigating it. Rather, the focus of bioengineers should be on improving the design and matching the stiffness properties of the skin-interfacing components in the CPAP mask structure (or other skin-contacting devices), so that they match those of the skin and underlying tissues, thereby postponing or minimizing the potency of the cascade demonstrated here (Gefen et al. 2020).

To summarize, we developed a new cell-scale modeling framework of the damage progression in DRPUs which visualized and demonstrated the nonlinearity of cell damage transition from deformation-induced to inflammatoryrelated damage and how the viability of tissues confined between a rigid medical device (CPAP mask) and bone (skull) surfaces may be compromised. Noteworthy is that the present scenario of a MDRPU which develops under a CPAP mask is one example which can be extrapolated to many other clinically relevant scenarios, such as lip tissues under an endotracheal tube (Amrani and Gefen 2020), finger or toe tissues under a pulse oximeter, chin or occipital protuberance tissues under a cervical collar, forehead tissues compressed onto an operating table pad (Peko et al. 2020), under penile compression clamps (Levy et al. 2017a) and so on and so forth. The visualization and quantitative description of the damage cascade in MDRPUs are important not only from a basic science perspective, but also for creating the awareness among clinicians as well as industry and regulators with regards to the need for improving the design of skin-contacting medical devices (Gefen et al. 2020). The goal of revised device designs aimed at mitigating MDRPUs is to lower strain and stress exposures in skin and underlying soft tissues to reduce the deformation-inflicted cell damage and thereby eliminate or minimize the consequent inflammatory edema reaction that escalates the cell and tissue damage.
Acknowledgements This work was partially supported by the Israeli Ministry of Science \& Technology (Medical Devices Program Grant no. 3-17421, awarded to Professor Amit Gefen in 2020).

Funding This work was partially supported by the Israeli Ministry of Science \& Technology (Medical Devices Program Grant no. 3-17421, awarded to Professor Amit Gefen in 2020).

Data availability The data that support the findings of this study are available from the corresponding author $(\mathrm{AG})$ upon a reasonable request.

\section{Compliance with ethical standards}

Conflict of interest The authors declare that they have no conflict of interest.

\section{References}

Amrani G, Gefen A (2020) Which endotracheal tube location minimises the device-related pressure ulcer risk: the centre or a corner of the mouth? Int Wound J 17:268-276

Bader DL, Worsley PR, Gefen A (2019) Bioengineering considerations in the prevention of medical device-related pressure ulcers. Clin Biomech 67:70-77

Ben-Or Frank M, Shoham N, Benayahu D, Gefen A (2015) Effects of accumulation of lipid droplets on load transfer between and within adipocytes. Biomech Model Mechanobiol 14:15-28

Black J, Alves P, Brindle CT et al (2015) Use of wound dressings to enhance prevention of pressure ulcers caused by medical devices. Int Wound J 12:322-327

Breuls RGM, Sengers BG, Oomens CWJ et al (2002) Predicting local cell deformations in engineered tissue constructs: a multilevel finite element approach. J Biomech Eng 124:198-207

Comley K, Fleck NA (2010) A micromechanical model for the Young's modulus of adipose tissue. Int J Solids Struct 47:2982-2990

Gefen A (2018) The future of pressure ulcer prevention is here: detecting and targeting inflammation early. EWMA J 19:7-13

Gefen A (2019) How medical engineering has changed our understanding of chronic wounds and future prospects. Med Eng Phys $72: 13-18$

Gefen A (2020) The progress in etiological research of pressure ulcers : a spotlight on the complex relations between cell damage due to sustained tissue deformations and pain. Finnish J Wound Care 2:16-19

Gefen A, Ousey K (2020) Update to device-related pressure ulcers: SECURE prevention. COVID-19, face masks and skin damage. J Wound Care 29:245-259

Gefen A, Weihs D (2016) Cytoskeleton and plasma-membrane damage resulting from exposure to sustained deformations: a review of the mechanobiology of chronic wounds. Med Eng Phys 38:828-833

Gefen A, van Nierop B, Bader DL, Oomens CW (2008) Strain-time cell-death threshold for skeletal muscle in a tissue-engineered model system for deep tissue injury. J Biomech 41:2003-2012

Gefen A, Brienza D, Edsberg L et al (2019) The Etiology of Pressure Injuries. In: Prevention and Treatment of Pressure Ulcers/Injuries: Clinical Practice Guideline European Pressure Ulcer Advisory Panel (EPUAP), National Pressure Injury Advisory Panel (NPIAP) and the Pan Pacific Pressure Injury Al, 3rd edn.

Gefen A, Alves P, Ciprandi G et al (2020) Device-related pressure ulcers: SECURE prevention. J Wound Care 29:S1-S52 
Husmann MJ, Barton M, Amann-Vesti BR, Franzeck UK (2006) Postural effects on interstitial fluid pressure in humans. J Vasc Res 43:321-326

Katzengold R, Shoham N, Benayahu D, Gefen A (2015) Simulating single cell experiments in mechanical testing of adipocytes. Biomech Model Mechanobiol 14:537-547

Katzengold R, Topaz M, Gefen A (2016) Tissue loads applied by a novel medical device for closing large wounds. J Tissue Viability 25:32-40

Leopold E, Gefen A (2013) Changes in permeability of the plasma membrane of myoblasts to fluorescent dyes with different molecular masses under sustained uniaxial stretching. Med Eng Phys 35:601-607

Levy A, Kopplin K, Gefen A (2015) Adjustability and adaptability are critical characteristics of pediatric support surfaces. Adv Wound Care 4:615-622

Levy A, Fader M, Bader D, Gefen A (2017a) Penile compression clamps: a model of the internal mechanical state of penile soft tissues. Neurourol Urodyn 36:1645-1650

Levy A, Kopplin K, Gefen A (2017b) Device-related pressure ulcers from a biomechanical perspective. J Tissue Viability 26:57-68

Linder-Ganz E, Engelberg S, Scheinowitz M, Gefen A (2006) Pressuretime cell death threshold for albino rat skeletal muscles as related to pressure sore biomechanics. J Biomech 39:2725-2732

Michel CC, Curry FE (1999) Microvascular permeability. Physiol Rev 79(3):703-761

Peko L, Barakat-Johnson M, Gefen A (2020) Protecting prone positioned patients from facial pressure ulcers using prophylactic dressings: a timely biomechanical analysis in the context of the COVID-19 pandemic. Int Wound J 17(6):1595-1606

Peko Cohen L, Ovadia-Blechman Z, Hoffer O, Gefen A (2019) Dressings cut to shape alleviate facial tissue loads while using an oxygen mask. Int Wound J 16:813-826

Shoham N, Girshovitz P, Katzengold R et al (2014) Adipocyte stiffness increases with accumulation of lipid droplets. Biophys $\mathbf{J}$ 106:1421-1431

Shoham N, Mor-Yossef Moldovan L, Benayahu D, Gefen A (2015) Multiscale modeling of tissue-engineered fat: is there a deformation-driven positive feedback loop in adipogenesis? Tissue Eng-Part A 21:1354-1363

Shoham N, Levy A, Shabshin N et al (2017) A multiscale modeling framework for studying the mechanobiology of sarcopenic obesity. Biomech Model Mechanobiol 16:275-295

Slomka N, Gefen A (2012) Relationship between strain levels and permeability of the plasma membrane in statically stretched myoblasts. Ann Biomed Eng 40:606-618

Van Linthout S, Miteva K, Schöpe C (2014) Crosstalk between fibroblasts and inflammatory cells. Cardiovasc Res 102:258-269

Publisher's note Springer Nature remains neutral with regard to jurisdictional claims in published maps and institutional affiliations. 